\title{
Optimizing Dissolution Dynamic Nuclear Polarization
}

\author{
Aurélien Bornet ${ }^{\mathrm{a} *}$, Sami Jannin ${ }^{\mathrm{a}, \mathrm{b} *}$ \\ ${ }^{a}$ Institut des Sciences et Ingénierie Chimiques, Ecole Polytechnique Fédérale de Lausanne (EPFL), Batochime, CH-1015 Lausanne, Switzerland \\ ${ }^{b}$ Bruker BioSpin AG, Industriestrasse 26, 8117 Fällanden, Switzerland
}

\section{A R T I C L E I N F O}

\section{Contents}

1. Introduction

A B S T R A C T

This article is a short review of some of our recent developments in dissolution dynamic nuclear polarization (d-DNP). We present the basic principles of d-DNP, and motivate our choice to step away from conventional approaches. We then introduce a modified d-DNP recipe that can be summed up as follows:

(i) Using broad line polarizing agents to efficiently polarize ${ }^{1} \mathrm{H}$ spins

(ii) Increasing the magnetic field to $6.7 \mathrm{~T}$ and above.

(iii) Applying microwave frequency modulation.

(iv) Applying ${ }^{1} \mathrm{H}-{ }^{13} \mathrm{C}$ cross polarization.

(v) Transferring hyperpolarized solution through a magnetic tunnel.

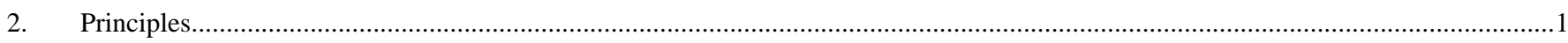

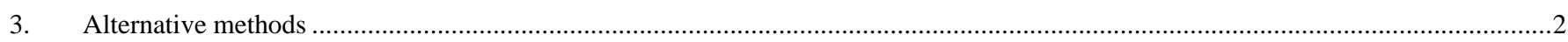

4. On-going developments ......................................................................................................... Error! Bookmark not defined.

\section{Introduction}

The idea of Dynamic Nuclear Polarization (DNP) is to transfer the large Boltzmann polarization of electrons to coupled nuclei via microwave irradiation. This idea was first proposed by Overhauser [1] and validated by Carver and Slichter [2]. Dissolution-DNP (d-DNP) was invented in 2003 by Ardenkjaer-Larsen et al. [3]. It is centered on the remarkable idea that the nuclear polarization that builds up through DNP in a solid at low temperatures in a moderate magnetic field can survive a dramatic temperature jump and a phase transition to a liquid at room temperature. It was shown in the original publication of J.-H. Ardenkjaer Larsen et al. that the liquid sample with enhanced polarization (said to be "hyperpolarized") could be transferred to a solution-state NMR or MRI machine through low fields (possibly as low as the earth's magnetic field) while preserving a significant part of its polarization.

\section{Principles}

\section{DNP polarizer}

In a d-DNP experiment, DNP is usually performed on a static sample at fairly low temperatures in a moderate magnetic field. Indeed, most DNP polarizers actually run at temperatures of about $T=1 \mathrm{~K}$, in magnetic fields of about $B_{0}$ $=3.35 \mathrm{~T}$. Under such conditions, the electron spin polarization is close to unity, which is an optimal situation for generating a nuclear spin polarization that is as high as possible prior to dissolution. The three main parts of a DNP polarizer are its cryostat, where the sample is usually immersed in a superfluid helium bath, a superconducting 
magnet that provides a sufficient field to achieve optimal electron spin polarization $P(e)$, and a microwave irradiation device operating at a frequency suitable for the saturation of electron spin resonance (ESR) transitions. It is interesting to note that, in practice, the most limiting factor here is often the availability of microwave sources with sufficient power (typically, one needs about $100 \mathrm{~mW}$ ) at high frequencies. At the EPFL for example, we are equipped with home-built dDNP polarizers [4-6] operating with continuous-flow ${ }^{4} \mathrm{He}$ bath cryostats (designed and built at the Paul Scherrer Institute) providing stable sample temperatures in the range of $T=1.2$ to $4.2 \mathrm{~K}$. In opposition to cryostats with variable temperature inserts (VTI), our cryostat operates independently from the helium vessel of the NMR magnet, and can be inserted in wide-bore magnet $\left(B_{0}=3.35\right.$ or $6.7 \mathrm{~T}$ in our laboratory). At $B_{0}=3.35 \mathrm{~T}$, microwave irradiation is generated by an ELVA microwave source with a maximum power $P_{\mu \mathrm{w}}=400 \mathrm{~mW}$ and a frequency range $v_{\mu \mathrm{w}}=94 \mathrm{GHz} \pm$ $250 \mathrm{MHz}$. The frequency can easily be doubled to $188 \mathrm{GHz} \pm$ $500 \mathrm{MHz}$ with a D200 doubler from Virginia Diodes to deliver about $P_{\mu \mathrm{w}}=100 \mathrm{~mW}$ for operation at $B_{0}=6.7 \mathrm{~T}$.

\section{Low temperature ${ }^{13} \mathrm{C}$ DNP with free radicals}

Dissolution-DNP has been mainly developed for magnetic resonance imaging (MRI) applications. ${ }^{13} \mathrm{C}$ was chosen as a target nuclear spin because 1) carbon is present in almost all relevant molecules in biology, 2) isotope labeling is often affordable, 3) the thermal equilibrium background signal of ${ }^{13} \mathrm{C}$ in-vivo is below detection limit and therefore not interfering, in contrast to $\left.{ }^{1} \mathrm{H}, 4\right)$ quaternary carbons have relatively long $T_{1}$ 's in liquid state at room temperature after dissolution so that the hyperpolarization can survive on sufficiently long timescales to probe relevant bio-chemical processes. Trityl radicals, a class of radicals with narrow ESR lines, are especially suited for the direct polarization of ${ }^{13} \mathrm{C}$ $[3,7,8]$. The two key parameters that describe the DNP efficiency of a free radical (1) the maximum polarization $P\left({ }^{13} \mathrm{C}\right)$ achievable and $(2)$ the time constant $\tau_{\mathrm{DNP}}\left({ }^{13} \mathrm{C}\right)$ of the approach to the DNP equilibrium. At $T=1.2 \mathrm{~K}$ and $B_{0}=3.35$ $\mathrm{T}$, trityl has been shown to polarize carbon-13 up to $P\left({ }^{13} \mathrm{C}\right)=$ $36 \%$ with a typical build-up time constant $\tau_{\mathrm{DNP}}\left({ }^{13} \mathrm{C}\right)=2300 \mathrm{~s}$ [9] (Figure 1a). With such a build-up time, one has to irradiate microwaves for about $3 \mathrm{~h}$ to reach $95 \%$ of the maximum polarization. The throughput of polarized samples can be increased with a multiple-sample approach. The samples can be polarized simultaneously and subsequently dissolved at wish one after the other $[9,10]$.

\section{Dissolution and liquid state MRI and NMR applications}

Once the sample has been dissolved and transferred to a NMR or MRI machine, provided that a significant part of the hyperpolarization is preserved, d-DNP makes it possible in principle to enhance magnetic resonance signals by several orders of magnitude, typically $\varepsilon_{\mathrm{DNP}}=10^{2}$ to $10^{4}$. Unfortunately, the high nuclear magnetization is only available for a short time. This is probably the main drawback of d-DNP. It can be used either in a single or in several consecutive experiments using pulses with small nutation angles. As a result, one usually cannot employ standard NMR or MRI pulse sequences on hyperpolarized samples. The detection sequences often need to be adapted, or even reinvented, to conform to the single-shot requirement. D-DNP has rapidly found a 'killer' application in in vivo MRI. The high signal-to-noise ratio provided by dDNP for ${ }^{13} \mathrm{C}$ labeled molecules enables the observation of hyperpolarized $1-{ }^{13} \mathrm{C}$ pyruvate in humans shortly after infusion, and its enzymatic in-cello conversion into $1-{ }^{13} \mathrm{C}$ lactate by lactate dehydrogenase (LDH). The pyruvate uptake and its conversion is much more rapid in cancer cells than in healthy tissue [11], which opens new perspectives for cancer diagnosis, in particular for prostate cancer. D-DNP for example made it possible to detect tumours by localized ${ }^{13} \mathrm{C}$ spectroscopy in human patients [12]. Such clinical applications call for the development of DNP polarizers that are specially designed for clinical applications, fulfilling specific requirements such as sterility [9]. Although most peer-reviewed papers utilizing d-DNP are concerned with localized spectroscopy or imaging of tumours following the infusion of hyperpolarized $1-{ }^{13} \mathrm{C}$ pyruvate, the technology of $\mathrm{d}-\mathrm{DNP}$ can in principle be used in combination with a variety of other NMR or MRI experiments, using an extensive diversity of molecules, and addressing many other nuclear spins. One can cite amongst many other publications in-vitro or in-cell studies [13, 14], drug screening [15], and monitoring of chemical reactions including the detection of intermediates [16]. Further d-DNP NMR applications can be found in reference [17]. The coupling with ultrafast ${ }^{1} \mathrm{H}-{ }^{13} \mathrm{C}$ and ${ }^{1} \mathrm{H}_{-}{ }^{15} \mathrm{~N}$ HSQC [18] is probably the best approach to combine multidimensional NMR with the single-shot requirement of d-DNP.

One important concern in d-DNP experiments is that once dissolved, the hyperpolarization decays with the nuclear spinlattice relaxation time $T_{1}$. This lifetime is usually relatively short, on the order of seconds or minutes. The hyperpolarization is therefore partly dissipated during the transfer step, and during additional quality control checks that are sometimes required (radical filtration, and measurements of concentration, temperature, or $\mathrm{pH}$ ). The same issue also arises during the infusion of hyperpolarized molecules into the blood stream, during the time lag before reaching the targeted organs for in-vivo applications, as well as during the signal acquisition. Multiple strategies have been explored and developed in the last years for minimizing losses of polarization, such as the use of fast fluid transfer devices [19], the rapid elimination of the free radicals [20-22], or the storage of hyperpolarization in the form of long-lived states (LLS) [23-28].

\section{Alternative methods}

\section{Efficient proton DNP using broad ESR line nitroxide radicals}

Most d-DNP studies involve directly hyperpolarizing carbon13 with trityl radicals. A straightforward and low-cost alternative consists in using one of the widely available nitroxide radicals, such as TEMPO (2,2,6,6tetramethylpiperidine 1-oxyl) or TEMPOL (4-Hydroxy TEMPO). Unfortunately, because of their broad ESR lines, nitroxide radicals are poor agents for direct polarization of ${ }^{13} \mathrm{C}$ under the conditions of temperatures and magnetic fields (typically $T=1.2 \mathrm{~K}$ and $B_{0}=3.35 \mathrm{~T}$ ) that are often used for trityl. Direct ${ }^{13} \mathrm{C}$ polarization using nitroxide radicals typically yields disappointing levels near $P\left({ }^{13} \mathrm{C}\right)=10 \%$, though with relatively short build-up times of $\tau_{\mathrm{DNP}}\left({ }^{13} \mathrm{C}\right)=325$ s. However, nitroxide radicals are excellent ${ }^{1} \mathrm{H}$ DNP agents yielding typically $P\left({ }^{1} \mathrm{H}\right)=40 \%$ in record times $\tau_{\mathrm{DNP}}\left({ }^{1} \mathrm{H}\right)=70$ $\mathrm{s}$ at $1.2 \mathrm{~K}[29,30]$ (Figure $1 \mathrm{~b}$ ). The DNP build-up time 
constants and the maximal polarizations for ${ }^{1} \mathrm{H}$ and ${ }^{13} \mathrm{C}$ measured at $3.35 \mathrm{~T}$ at different temperatures are reported in Table 1.

a)

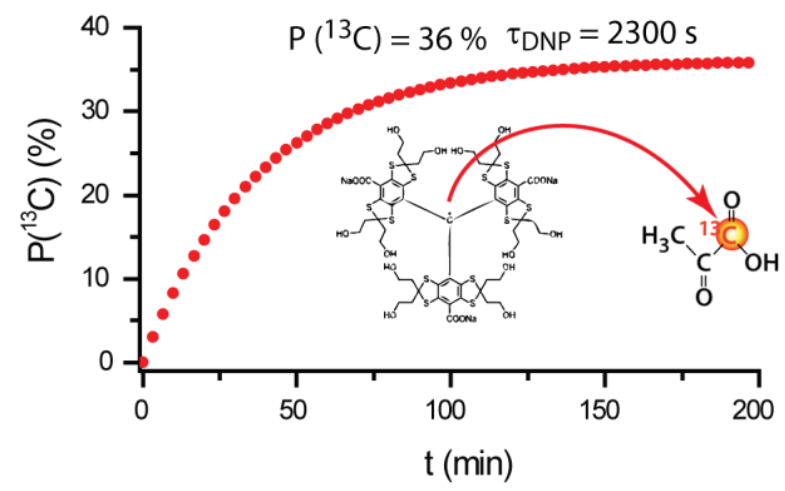

b)

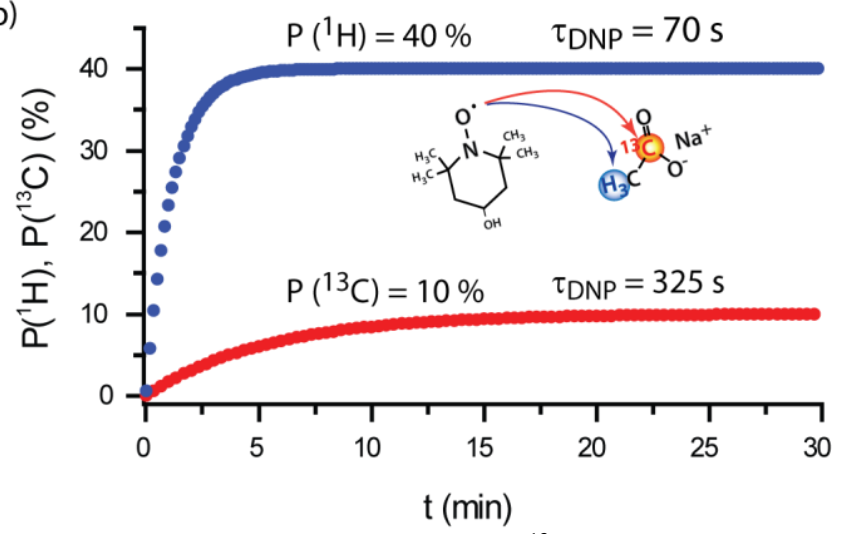

Figure 1: a) Typical DNP build-up of $1{ }^{13} \mathrm{C}$ pyruvic acid with 15 $\mathrm{mM}$ trityl at $1.2 \mathrm{~K}$ and $3.35 \mathrm{~T}$ [9] b) Typical ${ }^{1} \mathrm{H}$ (blue) and ${ }^{13} \mathrm{C}$ (red) DNP build-up curves of $3 \mathrm{M} 1{ }^{13} \mathrm{C}$ acetate with $30 \mathrm{mM}$ TEMPOL in $\mathrm{D}_{2} \mathrm{O}$ :glycerol- $\mathrm{d}_{8}(1: 1)$ at $1.2 \mathrm{~K}$ and $3.35 \mathrm{~T}[29,30]$.

\begin{tabular}{|c|c|c|c|c|c|}
\hline $\begin{array}{l}T \\
(K)\end{array}$ & $\begin{array}{c}P\left({ }^{1} \mathrm{H}\right) \\
(\%)\end{array}$ & $\begin{array}{c}\tau_{\mathrm{DNP}}\left({ }^{1} \mathrm{H}\right) \\
(\mathrm{s})\end{array}$ & $\begin{array}{c}P\left({ }^{13} \mathrm{C}\right) \\
(\%)\end{array}$ & $\begin{array}{c}\tau_{\mathrm{DNP}}\left({ }^{13} \mathrm{C}\right) \\
(\mathrm{s})\end{array}$ & $\varepsilon_{\mathrm{DNP}}\left({ }^{1} \mathrm{H} /{ }^{13} \mathrm{C}\right)$ \\
\hline 1.2 & 40 & 70 & 10 & 324 & 4 \\
\hline 2.2 & 24 & 57 & 6 & 267 & 4 \\
\hline 3.2 & 12 & 32 & 3 & 222 & 4 \\
\hline 4.2 & 8 & 22 & 2 & 158 & 4 \\
\hline
\end{tabular}

Table 1: ${ }^{1} \mathrm{H}$ and ${ }^{13} \mathrm{C}$ polarizations and build-up time constants in 3 M $1-{ }^{13} \mathrm{C}$ labeled sodium acetate with $30 \mathrm{mM}$ TEMPOL in $100 \%$ deuterated water:ethanol $(2: 1)$ at $B_{0}=3.35 \mathrm{~T}$ for different temperatures.

The ability of nitroxide radicals to rapidly and highly polarize high-gamma nuclear spins can be interesting on itself, since the hyperpolarization of ${ }^{1} \mathrm{H}$ and ${ }^{19} \mathrm{~F}$ can be used in many applications. One has to be aware that fast relaxation during transfer can be a severe drawback. However, several examples where d-DNP was used for high-gamma nuclear spins can be found in the literature. Fluorinated molecules are widely used in drug discovery for the detection of protein interactions, and ${ }^{19} \mathrm{~F}$ d-DNP provides a way of dramatically improving the sensitivity [15]. With an enhancement in the order of 1000, the detection of sub-micromolar concentrations of fluorinated binders was demonstrated. Hyperpolarized water can be used directly as a contrast agent for angiographic applications. High contrast-to-noise images can be obtained in a fraction of seconds [31]. In our laboratory, ${ }^{1} \mathrm{H}$ d-DNP was combined with long-lived proton states to enhanced both the sensitivity and contrast in drug screening experiments [32], and hyperpolarized water was used for protein-ligand interaction studies in combination with the so called WaterLOGSY technique [33].

\section{Improving DNP at higher magnetic fields}

At $B_{0}=3.35 \mathrm{~T}$ and $T=1.2 \mathrm{~K}$, electron spins are almost fully polarized $\left(P_{\mathrm{e}}=95 \%\right)$. It is therefore debatable if increasing the magnetic field could provide any improvement in DNP efficiency. Still, several studies at $2.5,4.5$ and $5 \mathrm{~T}$ with trityl $[34,35]$ and at $5 \mathrm{~T}$ with TEMPO [36] have clearly indicated that the nuclear spin polarization improves as the magnetic field increases, albeit at the price of longer build-up time constants. This time constraint becomes increasingly unreasonable for the hyperpolarization of low-gamma nuclei using trityl. For example, build-up time constants as long as $\tau_{\mathrm{DNP}}\left({ }^{13} \mathrm{C}\right)=95 \mathrm{~min}$ were measured at $5 \mathrm{~T}$ and $930 \mathrm{mK}$ using this polarizing agent [35]. On the other hand, DNP of highgamma nuclei $\left({ }^{1} \mathrm{H},{ }^{19} \mathrm{~F}\right)$ at high magnetic fields can improve the maximal polarization while the polarization times remain reasonably short. The main limitation or challenge remains to find microwave sources that are suitable for higher microwave frequencies. In our laboratory, the DNP polarizer used at $3.35 \mathrm{~T}$ was adapted by doubling the magnetic field to 6.7 $\mathrm{T}$ and the microwave frequency to $188 \mathrm{GHz}$, using a, superconducting magnet (Oxford Instruments) that was originally designed for $7.05 \mathrm{~T}$. The microwave source (ELVA) initially operating at $94 \mathrm{GHz}$ with $P^{\max }=400 \mathrm{~mW}$ was coupled to a frequency doubler (VDI/D200) to yield 188 $\mathrm{GHz}$ with an efficiency close to $25 \%$, leading to $P^{\max }=100$ $\mathrm{mW}$. Increasing the magnetic field leads to significant inhomogeneous broadening of the ESR lines of nitroxide radicals because of g-anisotropy. For reasons related to homogeneous broadening and spectral spin diffusion that are beyond the scope of this review, it was necessary to increase the radical concentrations to insure good DNP efficiency. At $T=1.2 \mathrm{~K}$, using a frozen glassy solution containing $50 \mathrm{mM}$ TEMPOL, a polarization $P\left({ }^{13} \mathrm{C}\right)=36 \%$ could be obtained directly with a long build-up time constant $\tau_{\mathrm{DNP}}\left({ }^{13} \mathrm{C}\right)=2000$ s. However, the proton polarization typically builds up to values as high as $P\left({ }^{1} \mathrm{H}\right)=90 \%$ in much shorter times $\tau_{\text {DNP }}\left({ }^{1} \mathrm{H}\right)=150 \mathrm{~s}$ (Figure 2)[37]. 


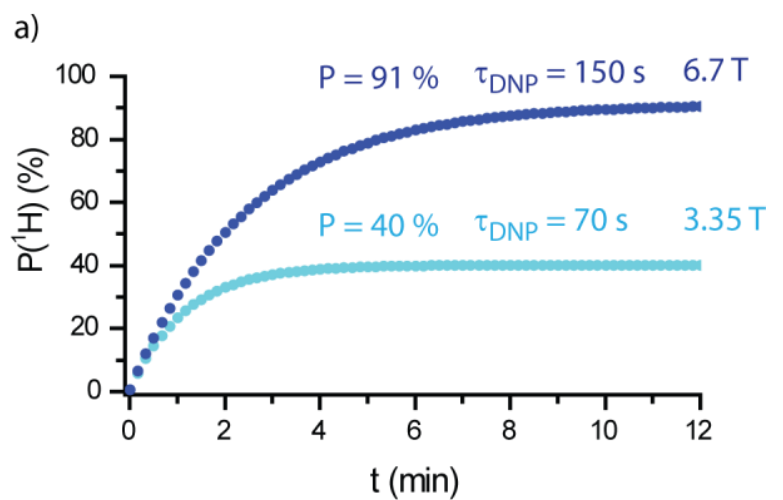

b)

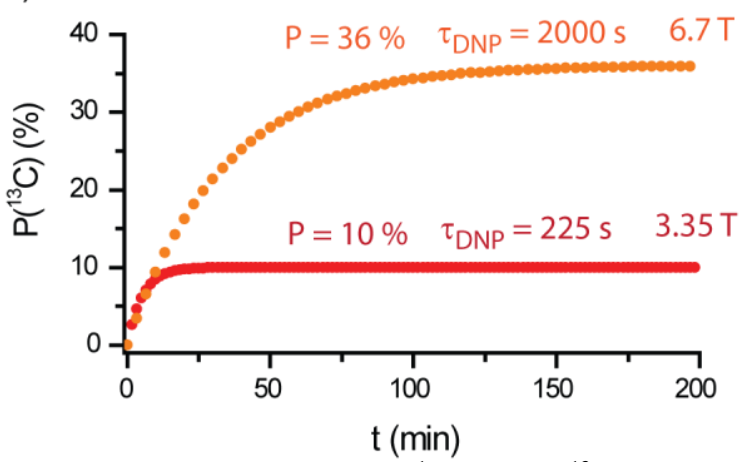

Figure 2: Comparison between ${ }^{1} \mathrm{H}$ (a) and ${ }^{13} \mathrm{C}$ (b) DNP build-up curves of $3 \mathrm{M} 1-{ }^{13} \mathrm{C}$ acetate in $\mathrm{D}_{2} \mathrm{O}$ : glycerol-d $\mathrm{d}_{8}(1: 1)$ at $1.2 \mathrm{~K}$ and $3.35 \mathrm{~T}$ (with $30 \mathrm{mM}$ TEMPOL) [29, 30] and $6.7 \mathrm{~T}(50 \mathrm{mM}$ TEMPOL) [37]. and $1.2 \mathrm{~K}$ and $6.7 \mathrm{~T}$ (with $50 \mathrm{mM}$ TEMPOL) [37].

The DNP build-up rates and maximal polarizations for ${ }^{1} \mathrm{H}$ and ${ }^{13} \mathrm{C}$ measured at $6.7 \mathrm{~T}$ at different temperatures are also reported in Table 2 .

\begin{tabular}{|l|l|l|l|l|l|}
\hline $\begin{array}{l}T \\
(\mathrm{~K})\end{array}$ & $\begin{array}{l}P\left({ }^{1} \mathrm{H}\right) \\
(\%)\end{array}$ & $\begin{array}{l}\tau_{\mathrm{DNP}}\left({ }^{1} \mathrm{H}\right) \\
(\mathrm{s})\end{array}$ & $\begin{array}{l}P\left({ }^{13} \mathrm{C}\right) \\
(\%)\end{array}$ & $\begin{array}{l}\tau_{\mathrm{DNP}}\left({ }^{13} \mathrm{C}\right) \\
(\mathrm{s})\end{array}$ & $\varepsilon_{\mathrm{DNP}}\left({ }^{1} \mathrm{H} /{ }^{13} \mathrm{C}\right)$ \\
\hline 1.2 & 91 & 150 & 36 & 1980 & 2.5 \\
\hline 2.2 & 60 & 52 & 22.5 & 1010 & 2.6 \\
\hline 4.2 & 25 & 25 & 5.5 & 359 & 4.5 \\
\hline
\end{tabular}

Table 2: ${ }^{1} \mathrm{H}$ and ${ }^{13} \mathrm{C}$ polarizations and corresponding build-up time constants of $3 \mathrm{M} 1-{ }^{13} \mathrm{C}$ labeled acetate with $50 \mathrm{mM}$ TEMPOL in $100 \%$ deuterated water:ethanol $(2: 1)$ at $B_{0}=6.7 \mathrm{~T}$ for different temperatures.

Polarization values $P\left({ }^{1} \mathrm{H}\right)$ close to unity are very important in the context of long-lived states (LLS). Levitt and coworkers [25] have shown that it is possible to populate LLS efficiently simply by achieving high polarizations, without any additional experimental manipulations such as $r f$ pulses, etc. The triplet-singlet imbalance (TSI) determines the expectation value of the available LLS. This imbalance is simply proportional to the square of the Zeeman polarization $\left(P_{\mathrm{TSI}}=-1 / 3 P_{\mathrm{Z}}^{2}\right)$.

\section{Boosting ${ }^{13}$ C DNP by cross polarization}

Apart from ${ }^{13} \mathrm{C},{ }^{19} \mathrm{~F}$ and ${ }^{1} \mathrm{H}$, d-DNP was successfully used to polarize other low-gamma nuclear spins, such as ${ }^{15} \mathrm{~N}$ [38], ${ }^{129} \mathrm{Xe}[39],{ }^{89} \mathrm{Y}[40,41]$, etc. These nuclei have the advantage of having long relaxation time constants at room temperature in the liquid-state, thus retaining most of their magnetization during dissolution and transfer. As shown before, trityl radicals can be very efficient as direct polarizing agents for ${ }^{13} \mathrm{C}$. Although such high polarization levels can provide intense NMR signals after dissolution, the long DNP build-up times $\tau_{\mathrm{DNP}}\left({ }^{13} \mathrm{C}\right)$ at $1.2 \mathrm{~K}$ do not allow one to perform several dissolution processes in rapid succession, as required for many in-vivo experiments with high throughput. Free radicals with broad ESR lines polarize nuclear spins with high gyromagnetic ratios such as ${ }^{1} \mathrm{H}$ and ${ }^{19} \mathrm{~F}$ very efficiently with short build-up times. As shown before, $P\left({ }^{1} \mathrm{H}\right)=40 \%$ and $\tau_{\mathrm{DNP}}\left({ }^{1} \mathrm{H}\right)=70 \mathrm{~s}$ at $1.2 \mathrm{~K}$ and $3.35 \mathrm{~T}[30]$ and $P\left({ }^{1} \mathrm{H}\right)$ can be as high as $90 \%$ with a build-up time constant of $150 \mathrm{~s}$ at $1.2 \mathrm{~K}$ and $6.7 \mathrm{~T}$ [37].

As will be shown below, the use of cross polarization (CP) [42-44] at low temperature in the DNP polarizer makes it possible to benefit from efficient DNP of high-gamma nuclear spins in the solid state, transfer their polarization to low-gamma nuclear spins, and take advantage of their long spin-lattice relaxation times after dissolution. This idea was already suggested in 1978 by Abragam and Goldman [45]: "The production of large polarization is more difficult for small nuclear moments. (...) Rare isotopes are also difficult to polarize; when the nuclear concentration is low, spin diffusion is slow which reduces considerably the rate of DNP and the limit that leakage relaxation sets for its maximum value. The situation is evidently even worse when nuclear moments are both rare and small. There are situations, however, when this drawback becomes an asset. This is when besides the rare isotope $S$, there exist in the sample an abundant nuclear species $I$, with a large $\gamma_{I}$, easily polarized by DNP. It is then possible, having first polarized the spin I by conventional DNP to transfer a part of the order they have acquired to the spin $S$ : in thermodynamic language, transfer some entropy from the spin $S$ to the spin I." Such a transfer of polarization is indeed possible with a well-established solidstate NMR method, the cross polarization (CP) technique. Cross polarization, along with Magic Angle Spinning (MAS), is the most widely used pulse scheme in solid state NMR. The original idea of Hartmann and Hahn of nuclear double resonance in the rotating frame [42] was adapted by Pines, Gibby and Waugh [43, 44] in view of enhancing NMR signals of dilute nuclear spins in solids. In such experiments, the large magnetization of a high-gamma, abundant nucleus (here ${ }^{1} \mathrm{H}$ ) is transferred to low-gamma, rare spins (here called $\mathrm{X})$. The standard $\mathrm{CP}$ procedure is the following. The ${ }^{1} \mathrm{H}$ spins are polarized in high $B_{0}$ field by allowing the Boltzmann equilibrium to be established (in our case, the ${ }^{1} \mathrm{H}$ spins are hyperpolarized to their steady-state DNP equilibrium). A $90^{\circ}$ excitation pulse followed by a phase-shifted spin-lock pulse with an $r f$ amplitude $B_{1}\left({ }^{1} \mathrm{H}\right)$ are then applied. The next step consists in establishing a contact between the ${ }^{1} \mathrm{H}$ and $\mathrm{X}$ spins to enable the transfer of polarization. To do so, a spin lock field of intensity $B_{1}(\mathrm{X})$ is simultaneously applied to the $\mathrm{X}$ spins. The contact between the two nuclear species is established if the so-called Hartmann-Hahn condition is fulfilled, i.e., if $\gamma\left({ }^{1} \mathrm{H}\right) B_{1}\left({ }^{1} \mathrm{H}\right)=\gamma(\mathrm{X}) B_{1}(\mathrm{X})$.

Cross polarization can thus be applied to boost the polarization of low-gamma nuclei. To do so, the CP method has to be implemented in the polarizer, i.e., in a cryostat at temperatures between 1.0 and $4.2 \mathrm{~K}$. The microwave irradiation must also to be applied to the sample. Finally, one should allow for dissolution. Thus, three hardware and software adaptations have to be done to render CP compatible with dissolution-DNP. A doubly resonant NMR coil needs to be designed to perform $\mathrm{CP}$ in the DNP polarizer. In our laboratory, we have first investigated the feasibility of low temperature $\mathrm{CP}$ with a probe optimized for DNP and CP but not compatible with dissolution. It consisted into a $0.1 \mathrm{cc}$ 
volume solenoidal coil tuned for ${ }^{1} \mathrm{H}$ and ${ }^{13} \mathrm{C}$ with $v\left({ }^{1} \mathrm{H}\right)=$ $142.57 \mathrm{MHz}$ and $v\left({ }^{13} \mathrm{C}\right)=35.85 \mathrm{MHz}$ at $B_{0}=3.35 \mathrm{~T}[29,30]$. This CP-DNP probe is shown in Figure 3a. To have an optimal quality factor, we decided to place the capacitors as close as possible to the coil. Fine tuning and matching of the circuit was sometimes necessary to ensure efficient $\mathrm{CP}$ transfer, and was performed with an external tuning and matching circuit. The whole NMR circuit was coupled to a standard Bruker solid-state NMR console. The same coil design optimized for DNP and CP was also adapted at $6.7 \mathrm{~T}$ [37]. d-DNP requires that the sample volume within the coil be easily and rapidly accessible from the top, in view of rapid dissolution of the sample after CP-DNP. The horizontal $0.1 \mathrm{cc}$ solenoidal coil design was thus replaced with a vertical $1 \mathrm{cc}$ saddle coil design [46]. As seen in Figure 3b, such a design enables simple insertion of a sample holder containing the sample into the accessible volume in the center the rf coils. The sample can be easily dissolved with a dissolution stick that can be coupled to the top of the sample holder. Such probe geometry with a saddle coil design and its large accessible volume inevitably lead to lower rf and microwave $B_{1}$ efficiencies than for the horizontal solenoidal coil design. (a)

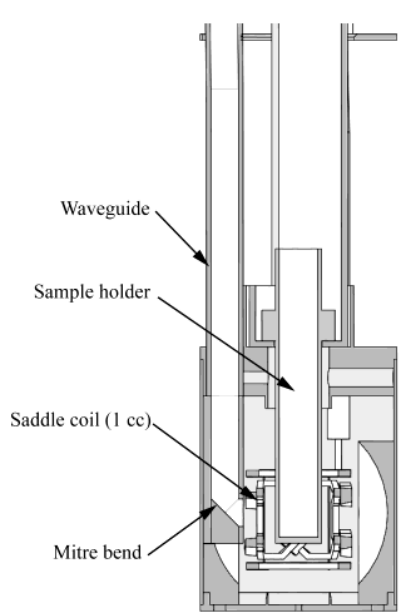

(b)

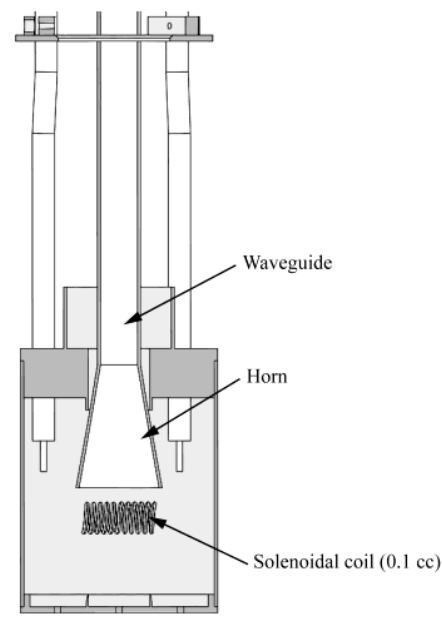

Figure 3: a) CP-DNP system with a saddle coil design for rapid dissolution. The sample holder can be inserted from the top into the accessible volume of the rf coils and is accessible for dissolution. b) CP-DNP system with a horizontal solenoid that is not suitable for rapid dissolution but that has more efficient microwave irradiation and cross-polarization capabilities.

The pulse sequence for cross polarization $(\mathrm{CP})$ needs to be adapted to the requirements of d-DNP (Figure 4). Unlike normal CP experiments, the goal here is not to observe enhanced ${ }^{13} \mathrm{C}$ signals in the polarizer, but to save longitudinal ${ }^{13} \mathrm{C}$ polarization, and to dissolve and transfer the sample prior to observation. Therefore, the ${ }^{13} \mathrm{C}$ magnetization should be aligned along the $B_{0}$ quantization axis at the end of the sequence, and not in the transverse plane. A $90^{\circ}$ flip-back pulse [47] can be added at the end of the sequence for this purpose. Moreover, since the carbon magnetization evolves slowly towards its DNP steady state, and since the proton DNP build-up is fast, multiple-contact CP transfers can be accumulated to build up the enhanced ${ }^{13} \mathrm{C}$ magnetization, bypassing the problem of the low $\mathrm{CP}$ efficiency due to $B_{1}$ fields that are typically weaker than the linewidth of the ${ }^{1} \mathrm{H}$ spectrum. The ${ }^{1} \mathrm{H}$ magnetization that remains after each $\mathrm{CP}$ step can be preserved by bringing it back along the z-axis with a $90^{\circ}$ flip-back pulse. Finally, in order to exploit the longitudinal ${ }^{13} \mathrm{C}$ polarization that has built up during the previous $\mathrm{CP}$ contacts, this longitudinal magnetization should be brought to the transverse plane before the $\mathrm{CP}$ contact, which can be achieved by the initial $90^{\circ}$ pulse on the ${ }^{13} \mathrm{C}$ channel in Figure 4. To monitor the build-up of the ${ }^{13} \mathrm{C}$ magnetization, small-angle pulses can be added between the CP steps.

Some parameters of the $\mathrm{CP}$ contact pulses have to be adjusted carefully. Under DNP conditions (in static solids at $1.2 \mathrm{~K}$ in the presence of paramagnetic species), $T_{1 \rho}$ is relatively short, typically on the order of a few milliseconds. Moreover, both the $r f$ field strength and the contact time must be limited to avoid arcing in the circuit, especially at pressures below 1 mbar where helium is in a superfluid state. An additional problem of CP under our conditions arises from the fact that both the $r f$ phase and the amplitude have to be switched between the $90^{\circ}$ excitation pulses and the cw contact pulses. The same switch occurs at the end of the contact pulses, before flipping the magnetization vectors back to the $\mathrm{z}$-axis. Under our DNP conditions at $1.2 \mathrm{~K}$, the switching delays (typically $4 \mu \mathrm{s}$ on a Bruker DRX console) are so long that they cause appreciable losses because of decoherence of transverse magnetizations. The $90^{\circ}$ excitation and flip-back pulses are also critical in standard $\mathrm{CP}$ sequences. Finally, the breadth of the ${ }^{1} \mathrm{H}$ spectrum is on the order of $50 \mathrm{kHz}$ for DNP samples at $3.35 \mathrm{~T}$ and $1.2 \mathrm{~K}$ (for the ${ }^{13} \mathrm{C}$ spectrum, it is on the order of $6 \mathrm{kHz}$ ). It is therefore a challenge to obtain a uniform excitation over the entire width of the lines. The CP sequence was therefore modified as follows: the standard pulse train $90^{\circ}{ }_{\mathrm{x}}$-contact $\mathrm{y}_{\mathrm{y}}-90^{\circ}{ }_{\mathrm{x}}$ was replaced by a scheme using an adiabatic half-passage or an adiabatic inversions [48].
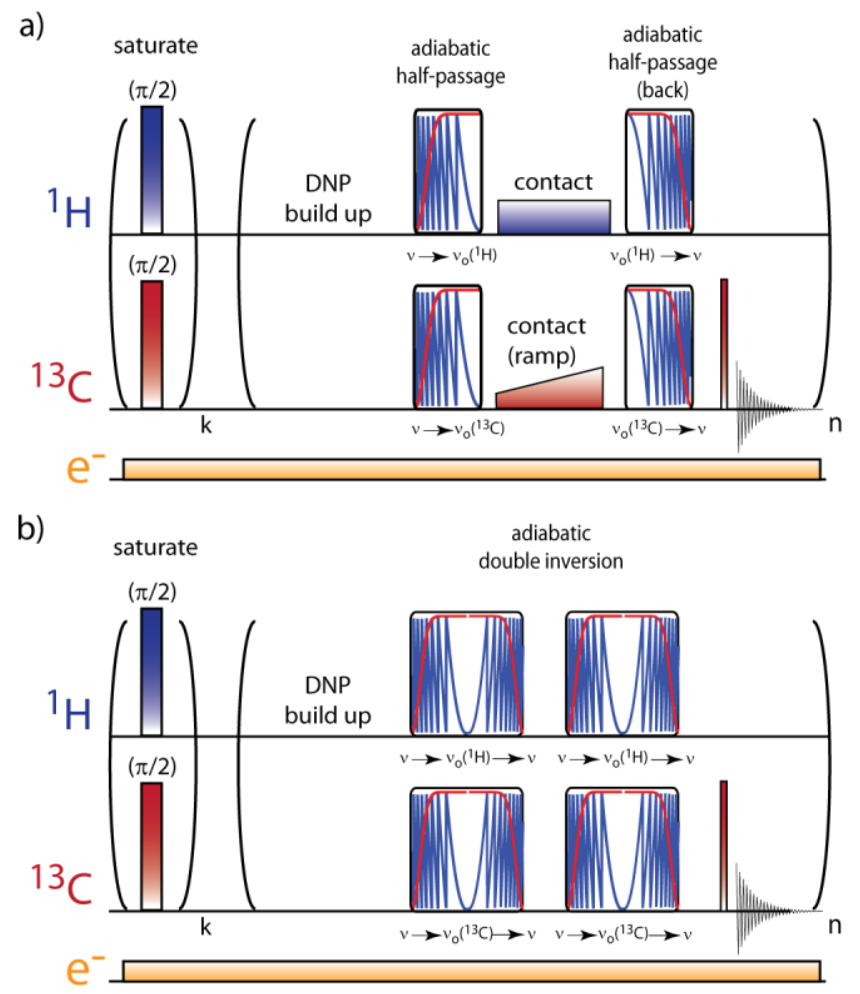

Figure 4: a) Multiple contact $\mathrm{CP}$ sequence using adiabatic halfpassage sweeps on both channels to excite and flip-back the ${ }^{1} \mathrm{H}$ and ${ }^{13} \mathrm{C}$ magnetizations, combined with $\mathrm{CW}$ contact pulses, the amplitude of which may be ramped. b) Multiple contact $\mathrm{CP}$ sequence where the magnetization is transferred during adiabatic inversion. The contact is established twice during each CP step. 
The most widely used version of frequency swept pulses is the hyperbolic secant [48]. A more broadband version known as WURST (which stands for "wideband, uniform rate and smooth truncation") was developed by Geen and Freeman [49]. We used the latter method for our DNP cross polarization experiments. Such swept pulses offer perfect solutions to the problems posed by standard CP sequences under DNP conditions. They can invert or excite a wide frequency range, and require less power than conventional "hard" pulses to be efficient. Finally, as the magnetization follows the effective field, no phase switch is necessary once the magnetization has reached the transverse plane. Such an adiabatic CP sequence is shown in Figure 4a. An adiabatic half-passage is performed on both ${ }^{1} \mathrm{H}$ and ${ }^{13} \mathrm{C}$ channels to bring the proton and carbon magnetization in the transverse plane. The contact pulse can be applied immediately after, without any switching of the $r f$ phase or amplitude. In Figure $4 \mathrm{a}$, a version is shown where an amplitude ramp is applied to the ${ }^{13} \mathrm{C}$ channel so that $\mathrm{CP}$ is less sensitive to $r f$ field inhomogeneities [50]. In practice, $50 \%$ to $100 \%$ amplitude ramps are used for optimal $\mathrm{CP}$ with our current probe. At the end of the contact, the magnetization is brought back to the $\mathrm{z}$ axes with two frequency-swept adiabatic half passage pulses from $v_{\mathrm{rf}}(\mathrm{t})=v_{0}(\mathrm{n})$ to $v_{\mathrm{rf}}(\mathrm{t})<<v_{0}(\mathrm{n})$. It is also possible to remove the contact pulses and to use only adiabatic fullpassage (AFP) pulses to perform cross polarization. To do so, the $r f$ field intensities have to be set on both channels so that the two nuclei are in contact in the frequency-modulated frame during the (AFP) pulse. As each pulse will invert the magnetization, a second $\mathrm{CP}$ contact has to be performed to store the magnetization along the $+\mathrm{z}$ axis between $\mathrm{CP}$ contacts (Figure 4b). Indeed, if only one CP contact is performed, the magnetization would be along the $-\mathrm{z}$ axis, and the DNP microwave irradiation would destroy the nuclear polarization, bringing the magnetization to a DNP steadystate with opposite sign. This method known as doubleinversion CP [51] is extremely convenient and robust to use.

The use of $\mathrm{CP}$ has the potential to increase the polarization from $P^{\mathrm{DNP}}\left({ }^{13} \mathrm{C}\right)$ to $P^{\mathrm{CP}-\mathrm{DNP}}\left({ }^{13} \mathrm{C}\right) \approx P^{\mathrm{DNP}}\left({ }^{1} \mathrm{H}\right)$, theoretically by a factor up to $\varepsilon_{\mathrm{CP}} \approx 4$ at $3.35 \mathrm{~T}$ and $1.2 \mathrm{~K}$, to a maximum absolute polarization $P^{\mathrm{CP}-\mathrm{DNP}}\left({ }^{13} \mathrm{C}\right)=40 \%$. Moreover, the use of CP allows one to obtain similar performances at $T>2.2 \mathrm{~K}$ than direct DNP at $1.2 \mathrm{~K}$, thus allowing the cryogenic equipment to be greatly simplified. The polarization levels $P^{\mathrm{CP}-\mathrm{DNP}}\left({ }^{13} \mathrm{C}\right)$ and $P^{\mathrm{DNP}}\left({ }^{13} \mathrm{C}\right)$ obtained for a $3 \mathrm{M}$ sample of 1 ${ }^{13} \mathrm{C}$ labeled acetate with $30 \mathrm{mM}$ TEMPOL in $100 \%$ deuterated water:ethanol $(2: 1)$ at different temperatures at $B_{0}$ $=3.35 \mathrm{~T}$ are shown in Table 3 [30]. With the solenoidal coil design used in our experiments, $\mathrm{CP}$ can provide an enhancement $\varepsilon_{\mathrm{CP}}>2$ compared to direct DNP. More importantly, the use of CP can also accelerate DNP build-up times by a factor $\kappa_{\mathrm{CP}}=2.6$ at $1.2 \mathrm{~K}$.

\begin{tabular}{|l|l|l|l|l|}
\hline$T(\mathrm{~K})$ & $P^{\mathrm{DNP}}\left({ }^{1} \mathrm{H}\right)(\%)$ & $P^{\mathrm{DNP}}\left({ }^{13} \mathrm{C}\right)(\%)$ & $\begin{array}{l}P^{\mathrm{DNP}-\mathrm{CP}}\left({ }^{13} \mathrm{C}\right) \\
(\%)\end{array}$ & $\varepsilon_{\mathrm{CP}}$ \\
\hline 1.2 & 40 & 10 & 23.0 & 2.3 \\
\hline 2.2 & 24 & 6 & 14.6 & 2.4 \\
\hline 3 & 12 & 3 & 7.2 & 2.4 \\
\hline 4.2 & 8 & 2 & 4.4 & 2.1 \\
\hline
\end{tabular}

Table 3: Maximal ${ }^{1} \mathrm{H}$ and ${ }^{13} \mathrm{C}$ polarizations achieved either by direct DNP or by CP-DNP with our solenoidal coil for $3 \mathrm{M} 1-{ }^{13} \mathrm{C}$ labeled acetate with $30 \mathrm{mM}$ TEMPOL in $100 \%$ deuterated water:ethanol (2:1) for different temperatures at $B_{0}=3.35 \mathrm{~T}$.

In this context, obtaining a high ${ }^{1} \mathrm{H}$ polarization in a short build-up time at $6.7 \mathrm{~T}$ is ideal for $\mathrm{CP}$. This was first tested at 6.7 $\mathrm{T}$ using a solenoid coil design that is not suitable for rapid dissolution. However, by implementing the multiple-contact $\mathrm{CP}$ sequence shown in Figure $4 \mathrm{~b}$, it was possible to achieve an unprecedented polarization level $P^{\mathrm{CP}-\mathrm{DNP}}\left({ }^{13} \mathrm{C}\right)=71 \%$ within a record build-up time $\tau_{\mathrm{CP}-\mathrm{DNP}}\left({ }^{13} \mathrm{C}\right)=490 \mathrm{~s}$ [37]. Thus, in addition to the gain in polarization, a significant acceleration of the build-up time constant was obtained with CP. With our approach, the DNP build-up time of the polarization is no longer a limiting factor for dissolutionDNP experiments. It can be seen in Table 4 that high ${ }^{13} \mathrm{C}$ polarizations can also be obtained at temperatures above 2.2 $\mathrm{K}$ with remarkably short build-up time constants, allowing for high-throughput high-sensitivity experiments with simplified cryogenic equipment.

\begin{tabular}{|l|l|l|l|l|l|l|l|}
\hline $\begin{array}{l}T \\
(\mathrm{~K})\end{array}$ & $\begin{array}{l}P^{\mathrm{DNP}}\left({ }^{1} \mathrm{H}\right) \\
(\%)\end{array}$ & $\begin{array}{l}P^{\mathrm{DNP}}\left({ }^{13} \mathrm{C}\right) \\
(\%)\end{array}$ & $\begin{array}{l}P^{\mathrm{DNP}-} \\
{ }_{\mathrm{CP}}\left({ }^{13} \mathrm{C}\right) \\
(\%)\end{array}$ & $\varepsilon_{\mathrm{CP}}$ & $\begin{array}{l}\tau_{\mathrm{DNP}}\left({ }^{13} \mathrm{C}\right) \\
(\mathrm{s})\end{array}$ & $\begin{array}{l}\tau_{\mathrm{CP}}\left({ }^{13} \mathrm{C}\right) \\
(\mathrm{s})\end{array}$ & $\kappa_{\mathrm{CP}}$ \\
\hline 1.2 & 91 & 36 & 71 & 2.0 & 1980 & 488 & 4.0 \\
\hline 2.2 & 60 & 22.5 & 43.8 & 2.0 & 1010 & 192 & 5.2 \\
\hline 4.2 & 25 & 5.5 & 15.5 & 2.8 & 359 & 70 & 5.1 \\
\hline
\end{tabular}

Table 4: Maximal ${ }^{1} \mathrm{H}$ and ${ }^{13} \mathrm{C}$ polarizations reached by CP-DNP with our solenoidal coil design for $3 \mathrm{M} 1-{ }^{13} \mathrm{C}$ labeled acetate with $50 \mathrm{mM}$ TEMPOL in $100 \%$ deuterated water:ethanol $(2: 1)$ at $B_{0}=6.7 \mathrm{~T}$ at different temperatures. The time constants of the direct DNP and CP-DNP of ${ }^{13} \mathrm{C}$ build-up curves as well as the corresponding acceleration factors $\kappa_{\mathrm{CP}}$ are also reported.

In order to make CP-DNP compatible with dissolution-DNP, we replaced the solenoidal coil by Helmholtz coils (Figure $3 b)$. The waveguide that in our initial design was terminated by a horn right above the sample was placed on the side of the cavity in our new design. The probe was tested on a sample of $3 \mathrm{M} 1{ }^{13} \mathrm{C}$ acetate dissolved in $\mathrm{D}_{2} \mathrm{O}$ :ethanol- $\mathrm{d}_{6}$ $(2: 1)$ with $50 \mathrm{mM}$ TEMPOL. With such a coil design, $P^{\text {DNP- }}$ ${ }^{\mathrm{CP}}\left({ }^{13} \mathrm{C}\right)=45 \%$, i.e., an enhancement due to $\mathrm{CP}$ of $\varepsilon_{\mathrm{CP}}=1.7$, could be obtained in a short build-up time $\tau_{\mathrm{CP}}\left({ }^{13} \mathrm{C}\right)=810 \mathrm{~s}$. The sample was then dissolved with $5 \mathrm{~mL}$ of superheated $\mathrm{D}_{2} \mathrm{O}$ and transferred in $10 \mathrm{~s}$ to a $7.05 \mathrm{~T}$ NMR spectrometer for detection. The room temperature signal was estimated to correspond to a remaining polarization $P\left({ }^{13} \mathrm{C}\right) \approx 40 \%$ (See Figure 5). Exactly the same experiment was applied to ${ }^{13} \mathrm{C}$ enriched sodium pyruvate and yielded $P\left({ }^{13} \mathrm{C}\right)=40.5 \%$ after dissolution [46].

a)

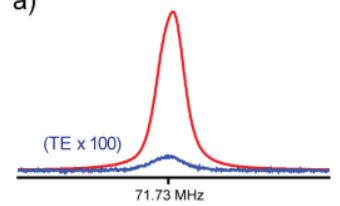

b)

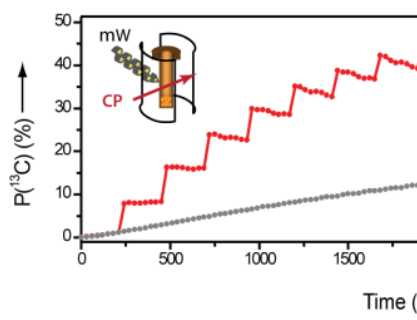

Figure 5: a) ${ }^{13} \mathrm{C}$ signals of a frozen $3 \mathrm{M}$ solution of $1-{ }^{13} \mathrm{C}$ enriched sodium acetate in a deuterated water/ethanol mixture $(67: 33 \mathrm{v} / \mathrm{v})$ doped with $50 \mathrm{mM}$ TEMPO measured observed at $T=4.2 \mathrm{~K}$ and $B_{0}$ $=6.7 \mathrm{~T}$ in $50 \mu \mathrm{L}$ : either using direct $\mathrm{DNP}$ without $\mathrm{CP}$ (blue) 
compared with a thermal equilibrium signal (scaled by a factor 100), or with CP-DNP (red) (with $P_{\mu \mathrm{w}}=120 \mathrm{~mW}$ at $v_{\mu \mathrm{w}}=188.3 \mathrm{GHz}$ ). b)

${ }^{13} \mathrm{C}$ DNP build-up curves using CP-DNP (red) or direct DNP (grey) without $\mathrm{CP}$, measured every $30 \mathrm{~s}$ with $5^{\circ}$ pulses. Multiple-contact $\mathrm{CP}$ was performed every $240 \mathrm{~s}$ with the adiabatic pulse sequence described in Figure $4 \mathrm{~b}$ with $B_{1}\left({ }^{1} \mathrm{H}\right)=B_{1}\left({ }^{13} \mathrm{C}\right)=15 \mathrm{kHz}$, and a contact time of $1 \mathrm{~ms}$. c) The dissolution and transfer process requires $t_{\text {diss }}=10.7 \mathrm{~s}$. d) Relaxation of ${ }^{13} \mathrm{C}$ in solution at $\mathrm{ca} . T=300$ $\mathrm{K}$ after transfer to a magnetic field $B_{0}=7.05 \mathrm{~T}$, measured with $5^{\circ}$ pulses at $5 \mathrm{~s}$ intervals. e) Signals of $1-{ }^{13} \mathrm{C}$ sodium acetate immediately after dissolution (red) and at thermal equilibrium (blue), scaled by a factor 2048 .

In the context of d-DNP, the use of $\mathrm{CP}$ is not limited to the transfer of polarization from ${ }^{1} \mathrm{H}$ to ${ }^{13} \mathrm{C}$, but can also be applied to other low-gamma nuclei. At the time of writing, CP-DNP probes designed for ${ }^{6} \mathrm{Li},{ }^{29} \mathrm{Si},{ }^{129} \mathrm{Xe}$ and ${ }^{15} \mathrm{~N}$ have been built in our laboratory. These nuclei have been so far less frequently addressed by d-DNP, not because the scope of their applications is narrower, but because, if one does not use $\mathrm{CP}$, their long build-up times and low polarization values have been two limiting factors. They appear promising since they exhibit long $T_{1}$ 's. For example $T_{1}\left({ }^{15} \mathrm{~N}\right)=800 \mathrm{~s}$ was measured in ${ }^{15} \mathrm{~N}$-enriched $\mathrm{d}_{9}$-trimethyl-phenyl-ammonium [52]. Finally, as discussed before, so-called long-lived states (LLS) can be populated directly if the polarization is very high [25], which can be readily achieved by CP under DNP conditions. We could thus envisage the use of CP-DNP to populate LLS in systems comprising pairs of ${ }^{15} \mathrm{~N}$ spins [26] or pairs of ${ }^{13} \mathrm{C}$ spins $[24,27,28]$.

\section{Applying microwave frequency modulation}

The use of paramagnetic polarizing agents is paradoxical along the course of a d-DNP experiment in the sense that (i) during the DNP build-up process they are mandatory (unpaired electrons are the source of polarization), but during dissolution, transfer and detection they are responsible for additional losses of polarization. The two main consequences are that the sensitivity is lessened and the time window available for measurement is shortened. This is particularly exacerbated for high-gamma nuclei like ${ }^{1} \mathrm{H}$. The presence of polarizing agents is also detrimental for cross polarization in the DNP polarizer as it broadens NMR line-widths and shortens $T_{1 \rho}$. Moreover, ${ }^{13} \mathrm{C}$ relaxation between $\mathrm{CP}$ steps is also enhanced by the presence of polarizing agents thus reducing the final ${ }^{13} \mathrm{C}$ polarization achievable. In a word, a reduction in their concentration would be very beneficial.

Nevertheless, the polarizing agents concentration is usually optimized for maximized DNP efficiency. A high concentration enhances electron-electron dipolar couplings, enabling rapid spectral spin diffusion within broad inhomogeneous ESR lines (especially true for nitroxides). As a consequence, a larger fraction of electron spins contribute to the DNP process. However, if the concentration is too high the ESR line tends to get homogeneously broadened beyond its inhomogeneous width, which leads to a reduced absolute DNP efficiency. A low concentration on the other hand leads to inefficient spectral spin diffusion. A reduced fraction of electron spins therefore contribute to DNP and therefore DNP build-up times become very long. In practice, the best concentration for nitroxide radicals was found to be around $50 \mathrm{mM}$ at $1.2 \mathrm{~K}$ and $6.7 \mathrm{~T}$.
In the d-DNP polarizer microwave irradiation of the ESR spectrum of polarizing agents is usually performed in a monochromatic fashion. It has been shown by Thurber et al.[53], Cassidy et al. [54] that DNP by CE and SE could be greatly improved by using either field modulation or microwave frequency modulation. More recently Hovav et al. [55] have shown that frequency modulation could improve $\mathrm{DNP}$ at $3.35 \mathrm{~T}$ in the temperature range $10-50 \mathrm{~K}$, close to the d-DNP conditions. We have recently shown that the same approach is also beneficial at lower temperatures $(T=1.2 \mathrm{~K})$ and at higher magnetic fields $\left(B_{0}=6.7 \mathrm{~T}\right)$ [56]. When microwave frequency modulation is applied, the DNP-active fraction of the ESR line is no longer determined solely by the polarizing agents concentration. In fact, frequency modulation somehow substitutes spectral spin diffusion. More detailed theoretical explanations supported by numerical simulations are given by Hovav et al. [55]. Modulating the microwave frequency can therefore generate competitive DNP enhancements, but with lower polarizing agents concentrations.

\section{Transferring hyperpolarized solutions through a magnetic tunnel}

As shown by Bryant and co-workers in studies of translational motion in the vicinity of paramagnetic nitroxides $[57,58]$, paramagnetic relaxation is extremely efficient at low field. This relaxation is particularly challenging in the context of d-DNP. Indeed, apart from the dual center magnet approach from Kockenberger [59] or the in-situ rapid melt approach from van Bentum [60], the hyperpolarized solution usually needs to be transferred to another magnet during which time it is exposed to very low magnetic fields (mT range). The exact magnetic field pattern experienced by the hyperpolarized fluid in our laboratory was mapped systematically with a triple-axis Hall probe [61]. The hyperpolarized solution effectively travels through a relatively low field, below $B_{0}=1 \mathrm{mT}$, with regions of close to zero-field, where the magnetic field gets inverted. 
a)

b)

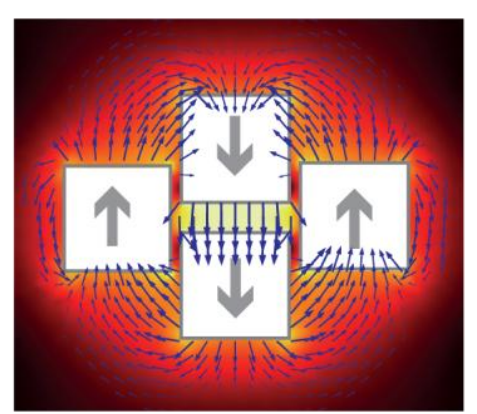

c)
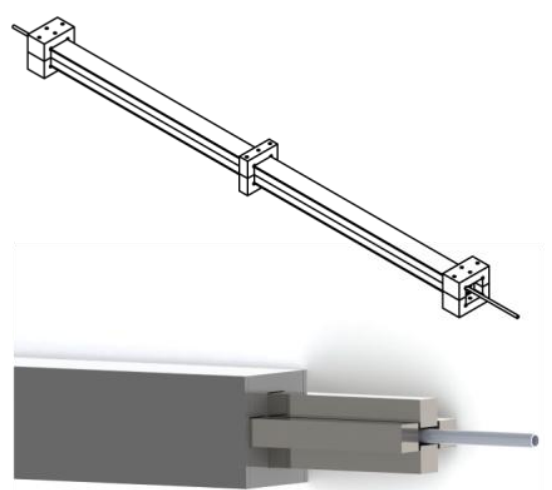

Figure 6: a) Simulations (Comsol Multiphysics) showing the magnetic field vectors in the magnetic tunnel where permanent magnets are positioned in four rows following a simplified Halbach design. b) Linear segment of $50 \mathrm{~cm}$ length. c) Schematic view of the permanent magnet arrangement around the PTFE tube maintained by the aluminum structure.

In order to protect hyperpolarized solutions from such low field environment and from the associated exacerbated paramagnetic relaxation, we have designed a modular magnetic tunnel to interface our polarizer either to an unshielded $300 \mathrm{MHz}$ or to an ultra-shielded $500 \mathrm{MHz}$ NMR spectrometer [61]. It consists of an assembly of permanent neodymium boron magnets securely maintained by several home built aluminum structures. The permanent magnets are positioned in four rows following a simplified Halbach design [62] and oriented to maximize the magnetic field strength in the center where $B_{\text {tunnel }}>0.9 \mathrm{~T}$ (Figure 6). A PTFE tube is inserted in the center of the aluminum structure to carry the hyperpolarized solution. Different modular segments comprising straight sections, bending sections with different angles and axial twists were designed. These segments can be assembled in a flexible way. The benefit of this magnetic tunnel had been illustrated by several examples with improvements factors ranging between $1<\varepsilon_{\text {tunnel }}<25$ for hyperpolarized proton, and to a lesser extent for carbon13.

\section{Conclusion}

We have described in this review a few methods for optimizing d-DNP that we have been developing during the last years in our laboratory. The modified d-DNP recipe that we present consists in using broad ESR line polarizing agents, increasing the magnetic field to $6.7 \mathrm{~T}$, and applying microwave frequency modulation, to polarize ${ }^{1} \mathrm{H}$ spins in a most efficient way. The high ${ }^{1} \mathrm{H}$ polarization is then transferred to ${ }^{13} \mathrm{C}$ spins by applying ${ }^{1} \mathrm{H}^{-1}{ }^{13} \mathrm{C}$ cross polarization.
Finally, the DNP sample is dissolved to room temperature and a magnetic tunnel is used to prevent exacerbated hyperpolarization losses at low fields during the voyage to the NMR or MRI machine. We showed how, by pushing the field up to $6.7 \mathrm{~T}$, one could obtain proton polarizations as high as $P\left({ }^{1} \mathrm{H}\right)=90 \%$ at $T=1.2 \mathrm{~K}$ and $P\left({ }^{1} \mathrm{H}\right)=60 \%$ at $T=2.2$ K. Competitive ${ }^{1} \mathrm{H}$ polarizations are therefore accessible at $T$ $=2.2 \mathrm{~K}$. By further increasing the magnetic field in the future, we are confident that competitive polarization levels will be affordable at even higher temperatures, e.g. $T=4.2 \mathrm{~K}$. The advantages of this strategy are that (i) one can avoid using complex and expensive cryogenic technology and that (ii) DNP build-up times become shorter at higher temperatures.

\section{Acknowledgments}

We would like to thank Prof. Geoffrey Bodenhausen for his supervision and constant and unconditional support on this dDNP project. His critical reading on the manuscript was greatly appreciated. The scientific content of this article builds on the work of numerous talented scientists in the team, amongst which Jonas Milani, Basile Vuichoud, Xiao Ji. We are indebt to Dr. Werner Maas, and Dr. Joost Lohman from Bruker BioSpin for actively supporting this research, and to Dr. Patrick Hautle and Dr. Ben van den Brandt from PSI for their cryogenic expertise. Martial Rey, Dr. Pascal Mieville and Anto Barisic from EPFL provided valuable technical assistance. The work was supported by the Swiss National Science Foundation (SNSF), the Ecole Polytechnique Fédérale de Lausanne (EPFL), the Swiss Commission for Technology and Innovation (CTI), Bruker BioSpin, the CNRS, and the European Research Council (ERC), Grant Agreement 339754 "Dilute para-water".

\section{References}

[1] A.W. Overhauser, Phys. Rev., 92 (1953) 411-415.

[2] T.R. Carver, C.P. Slichter, Phys. Rev., 92 (1953) 212-213. [3] J.H. Ardenkjaer-Larsen, B. Fridlund, A. Gram, G. Hansson, L. Hansson, M.H. Lerche, R. Servin, M. Thaning, K. Golman, Proc. Natl. Acad. Sci. U.S.A., 100 (2003) 1015810163.

[4] A. Comment, B. van den Brandt, K. Uffmann, F. Kurdzesau, S. Jannin, J.A. Konter, P. Hautle, W.T.H. Wenckebach, R. Gruetter, J.J. van der Klink, Concept. Magn. Reson. B, 31B (2007) 255-269.

[5] S. Jannin, Dynamic Nuclear Polarization Techniques for Magnetic Resonance Imaging and Particles Targets Experiments, in: Faculté des Sciences de Base, Ecole Polytechnique Fédérale de Lausanne, Lausanne, 2009.

[6] F. Kurdzesau, Some Methods of Dynamic Nuclear Polarization for Use in Metabolic Imaging, in: Faculté des Sciences de Base, Ecole Polytechnique Fédérale de Lausanne, Lausanne, 2009.

[7] J.H. Ardenkjaer-Larsen, S. Macholl, H. Johannesson, Appl. Magn. Reson., 34 (2008) 509-522.

[8] J. Wolber, F. Ellner, B. Fridlund, A. Gram, H. Johannesson, G. Hansson, L.H. Hansson, M.H. Lerche, S. Mansson, R. Servin, M. Thaning, K. Golman, J.H. Ardenkjaer-Larsen, Nucl. Instrum. Meth. A, 526 (2004) 173181.

[9] J.H. Ardenkjaer-Larsen, A.M. Leach, N. Clarke, J. Urbahn, D. Anderson, T.W. Skloss, NMR Biomed., 24 (2011) 927-932. 
[10] M. Batel, M. Krajewski, K. Weiss, O. With, A. Dapp, A. Hunkeler, M. Gimersky, K.P. Pruessmann, P. Boesiger, B.H. Meier, S. Kozerke, M. Ernst, J. Magn. Reson., 214 (2012) 166-174.

[11] J. Kurhanewicz, D.B. Vigneron, K. Brindle, E.Y. Chekmenev, A. Comment, C.H. Cunningham, R.J. DeBerardinis, G.G. Green, M.O. Leach, S.S. Rajan, R.R. Rizi, B.D. Ross, W.S. Warren, C.R. Malloy, Neoplasia, 13 (2011) 81-97.

[12] S.J. Nelson, J. Kurhanewicz, D.B. Vigneron, P.E.Z. Larson, A.L. Harzstark, M. Ferrone, M. van Criekinge, J.W. Chang, R. Bok, I. Park, G. Reed, L. Carvajal, E.J. Small, P. Munster, V.K. Weinberg, J.H. Ardenkjaer-Larsen, A.P. Chen, R.E. Hurd, L.I. Odegardstuen, F.J. Robb, J. Tropp, J.A. Murray, Sci. Transl. Med., 5 (2013).

[13] C. Cabella, M. Karlsson, C. Canape, G. Catanzaro, S.C. Serra, L. Miragoli, L. Poggi, F. Uggeri, L. Venturi, P.R. Jensen, M.H. Lerche, F. Tedoldi, J. Magn. Reson., 232 (2013) 45-52.

[14] S. Meier, M. Karlsson, P.R. Jensen, M.H. Lerche, J.O. Duus, Mol. Biosyst., 7 (2011) 2834-2836.

[15] Y. Lee, H.F. Zeng, S. Ruedisser, A.D. Gossert, C. Hilty, J. Am. Chem. Soc., 134 (2012) 17448-17451.

[16] P.R. Jensen, S. Meier, J.H. Ardenkjaer-Larsen, J.O. Duus, M. Karlsson, M.H. Lerche, Chem. Commun., (2009) 5168-5170.

[17] S. Meier, P.R. Jensen, M. Karlsson, M.H. Lerche, Sensors, 14 (2014) 1576-1597.

[18] L. Frydman, D. Blazina, Nat. Phys., 3 (2007) 415-419.

[19] S. Bowen, C. Hilty, Phys. Chem. Chem. Phys., 12 (2010) 5766-5770.

[20] D. Gajan, A. Bornet, B. Vuichoud, J. Milani, R. Melzi, H.A. van Kalkeren, L. Veyre, C. Thieuleux, M.P. Conley, W.R. Gruning, M. Schwarzwalder, A. Lesage, C. Coperet, G. Bodenhausen, L. Emsley, S. Jannin, Proc. Natl. Acad. Sci. U. S. A., 111 (2014) 14693-14697.

[21] T. Harris, C. Bretschneider, L. Frydman, J. Magn. Reson., 211 (2011) 96-100.

[22] P. Mieville, P. Ahuja, R. Sarkar, S. Jannin, P.R. Vasos, S. Gerber-Lemaire, M. Mishkovsky, A. Comment, R. Gruetter, O. Ouari, P. Tordo, G. Bodenhausen, Angew. Chem. Int. Edit., 49 (2010) 6182-6185.

[23] A. Bornet, X. Ji, D. Mammoli, B. Vuichoud, J. Milani, G. Bodenhausen, S. Jannin, Chem. Eur. J., 20 (2014) 1711317118.

[24] G. Pileio, S. Bowen, C. Laustsen, M.C.D. Tayler, J.T. Hill-Cousins, L.J. Brown, R.C.D. Brown, J.H. ArdenkjaerLarsen, M.H. Levitt, J. Am. Chem. Soc., 135 (2013) 50845088.

[25] M.C.D. Tayler, I. Marco-Rius, M.I. Kettunen, K.M. Brindle, M.H. Levitt, G. Pileio, J. Am. Chem. Soc., 134 (2012) 7668-7671.

[26] R.K. Ghosh, S.J. Kadlecek, J.H. Ardenkjaer-Larsen, B.M. Pullinger, G. Pileio, M.H. Levitt, N.N. Kuzma, R.R. Rizi, Magn. Reson. Med., 66 (2011) 1177-1180.

[27] C. Laustsen, G. Pileio, M.C.D. Tayler, L.J. Brown, R.C.D. Brown, M.H. Levitt, J.H. Ardenkjaer-Larsen, Magn. Reson. Med., 68 (2012) 1262-1265.

[28] I. Marco-Rius, M.C.D. Tayler, M.I. Kettunen, T.J. Larkin, K.N. Timm, E.M. Serrao, T.B. Rodrigues, G. Pileio, J.H. Ardenkjaer-Larsen, M.H. Levitt, K.M. Brindle, NMR Biomed., 26 (2013) 1696-1704.

[29] S. Jannin, A. Bornet, S. Colombo, G. Bodenhausen, Chem. Phys. Lett., 517 (2011) 234-236.
[30] A. Bornet, R. Melzi, S. Jannin, G. Bodenhausen, Appl. Magn. Reson., 43 (2012) 107-117.

[31] J.H. Ardenkjaer-Larsen, C. Laustsen, S. Bowen, R. Rizi, Magn. Reson. Med., 71 (2014) 50-56.

[32] R. Buratto, A. Bornet, J. Milani, D. Mammoli, B. Vuichoud, N. Salvi, M. Singh, A. Laguerre, S. Passemard, S. Gerber-Lemaire, S. Jannin, G. Bodenhausen, ChemMedChem, 9 (2014) 2509-2515.

[33] Q. Chappuis, J. Milani, B. Vuichoud, A. Bornet, A.D. Gossert, G. Bodenhausen, S. Jannin, J. Phys. Chem. Lett., 6 (2015) 1674-1678.

[34] H. Johanneson, S. Macholl, J.H. Ardenkjaer-Larsen, J. Magn. Reson., 197 (2009) 167-175.

[35] W. Meyer, J. Heckmann, C. Hess, E. Radtke, G. Reicherz, L. Triebwasser, L. Wang, Nucl. Instrum. Meth. A, 631 (2011) 1-5.

[36] S. Jannin, A. Comment, F. Kurdzesau, J.A. Konter, P. Hautle, B. van den Brandt, J.J. van der Klink, J. Chem. Phys., 128 (2008).

[37] S. Jannin, A. Bornet, R. Melzi, G. Bodenhausen, Chem. Phys. Lett., 549 (2012) 99-102.

[38] R. Sarkar, A. Comment, P.R. Vasos, S. Jannin, R. Gruetter, G. Bodenhausen, H. Hall, D. Kirik, V.P. Denisov, J. Am. Chem. Soc., 131 (2009) 16014.

[39] A. Comment, S. Jannin, J.N. Hyacinthe, P. Mieville, R. Sarkar, P. Ahuja, P.R. Vasos, X. Montet, F. Lazeyras, J.P. Vallee, P. Hautle, J.A. Konter, B. van den Brandt, J.P. Ansermet, R. Gruetter, G. Bodenhausen, Phys. Rev. Lett., 105 (2010).

[40] P. Mieville, S. Jannin, L. Helm, G. Bodenhausen, J. Am. Chem. Soc., 132 (2010) 5006.

[41] A.K. Jindal, M.E. Merritt, E.H. Suh, C.R. Malloy, A.D. Sherry, Z. Kovacs, J. Am. Chem. Soc., 132 (2010) 1784-+.

[42] S.R. Hartmann, E.L. Hahn, Phys. Rev., 128 (1962) 2042.

[43] A. Pines, M.G. Gibby, J.S. Waugh, J. Chem. Phys., 59 (1973) 569-590.

[44] A. Pines, J.S. Waugh, M.G. Gibby, J. Chem. Phys., 56 (1972) 1776.

[45] A. Abragam, M. Goldman, Rep. Prog. Phys., 41 (1978) 395-467.

[46] A. Bornet, R. Melzi, A.J. Perez-Linde, P. Hautle, B. van den Brandt, S. Jannin, G. Bodenhausen, J. Phys. Chem. Lett., 4 (2013) 111-114.

[47] J. Tegenfeldt, U. Haeberlen, J. Magn. Reson., 36 (1979) 453-457.

[48] J. Baum, R. Tycko, A. Pines, Phys. Rev. A, 32 (1985) 3435-3447.

[49] E. Kupce, R. Freeman, J. Magn. Reson., Ser. A, 115 (1995) 273-276.

[50] S. Hediger, B.H. Meier, N.D. Kurur, G. Bodenhausen, R.R. Ernst, Chem. Phys. Lett., 223 (1994) 283-288.

[51] A.J. Perez Linde, Application of Cross Polarization Techniques to Dynamic Nuclear Polarization Dissolution Experiments, in: School of Physics and Astromomy, University of Notthingham, Nottingham, 2010.

[52] H. Nonaka, R. Hata, T. Doura, T. Nishihara, K. Kumagai, M. Akakabe, M. Tsuda, K. Ichikawa, S. Sando, Nat. Commun., 4 (2013) 2411.

[53] K.R. Thurber, W.M. Yau, R. Tycko, J. Magn. Reson., 204 (2010) 303-313.

[54] M.C. Cassidy, H.R. Chan, B.D. Ross, P.K. Bhattacharya, C.M. Marcus, Nat. Nanotechnol., 8 (2013) 363-368.

[55] Y. Hovav, A. Feintuch, S. Vega, D. Goldfarb, J. Magn. Reson., 238 (2014) 94-105. 
[56] A. Bornet, J. Milani, B. Vuichoud, A.J.P. Linde, G. Bodenhausen, S. Jannin, Chem. Phys. Lett., 602 (2014) 6367.

[57] B. Borah, R.G. Bryant, J. Chem. Phys., 75 (1981) 32973300 .

[58] C.F. Polnaszek, R.G. Bryant, J. Chem. Phys., 81 (1984) 4038-4045.

[59] J. Leggett, R. Hunter, J. Granwehr, R. Panek, A.J. PerezLinde, A.J. Horsewill, J. McMaster, G. Smith, W. Kockenberger, Phys. Chem. Chem. Phys., 12 (2010) 58835892.

[60] M. Sharma, G. Janssen, J. Leggett, A.P.M. Kentgens, P.J.M. van Bentum, J. Magn. Reson., 258 (2015) 40-48.

[61] J. Milani, B. Vuichoud, A. Bornet, P. Mieville, R. Mottier, S. Jannin, G. Bodenhausen, Rev. Sci. Instrum., 86 (2015).

[62] K. Halbach, Nucl. Instrum. Methods., 169 (1980) 1-10. 